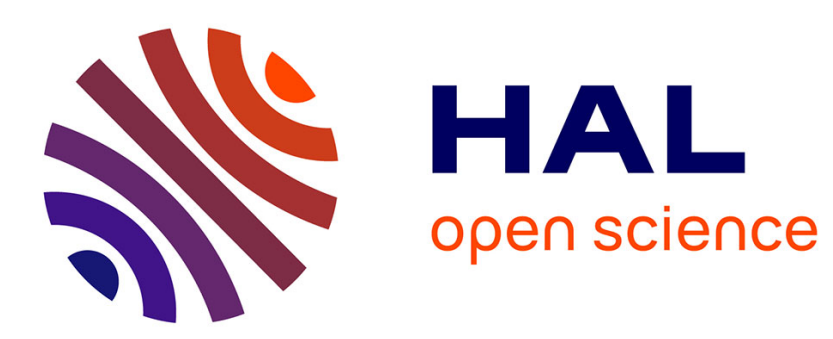

\title{
Note sur l'apparition des constructions à arases de briques dans la région lyonnaise
}

\author{
Armand Desbat
}

\section{To cite this version:}

Armand Desbat. Note sur l'apparition des constructions à arases de briques dans la région lyonnaise. Gallia - Fouilles et monuments archéologiques en France métropolitaine, 1992, 49, pp.45-50. 10.3406/galia.1992.2928 . hal-01915060

\section{HAL Id: hal-01915060 \\ https://hal.science/hal-01915060}

Submitted on 19 Jan 2020

HAL is a multi-disciplinary open access archive for the deposit and dissemination of scientific research documents, whether they are published or not. The documents may come from teaching and research institutions in France or abroad, or from public or private research centers.
L'archive ouverte pluridisciplinaire HAL, est destinée au dépôt et à la diffusion de documents scientifiques de niveau recherche, publiés ou non, émanant des établissements d'enseignement et de recherche français ou étrangers, des laboratoires publics ou privés.

\section{(이) $\$$}

Distributed under a Creative Commons Attribution - NonCommercial - NoDerivatives| 4.0 


\title{
Note sur l'apparition des constructions à arases de briques dans la région lyonnaise
}

\author{
par Armand DESBAT
}

\begin{abstract}
L'emploi d'arases de briques dans les constructions gallo-romaines a été souvent utilisé comme un indice ch II ${ }^{\mathrm{e}} \mathrm{E}$. dya du Gier) est actuellement remise en cause tandis que d'autres exemples dans la région lyonnaise attestent 1 usage de cette technique dès le début du I $^{\mathrm{er}} \mathrm{s}$.
\end{abstract}

The use of brick raws in roman buildings has been often considered as a good dating mark. The appearing of this tecinique in Gaule has been dated, until recenlly, from the beginning of the 2nd C. A.D. Yet, the chronology of some buiidings which have often been used as references, like the "Aqueduc du Gier", is now disputed, and other examples from the region show the use of this technique since the beginning of the 1st C. A.D.

Mots clés : rangs de briques, aqueduc du Gier, sanctuaire de Cybèle, Lyon, Rhône.

La rareté du matériel dateur, en particulier de la céramique, dans les édifices publics a souvent conduit à rechercher d'autres éléments de chronologie, notamment à utiliser les modes de construction pour dater ces monuments. Parmi ces modes de construction, l'emploi de chaînages de briques dans les murs de petit appareil a été souvent considéré comme un bon indice chronologique.

Deux publications relativement récentes viennent d'évoquer la question de la chronologie des constructions à arases de briques et de proposer de situer au II $^{\mathrm{e}} \mathrm{s}$. l'apparition de ce mode de construction en Gaule.
Dans la première, J.-P. Adam (1984, p. 151-156) fait de la tour de Vésone à Périgueux, dont l'édification se placerait sous le règne de Trajan, l'édifice le plus ancien en Gaule à avoir utilisé ce mode de construction. Dans la seconde, A. Audin (1985), à propos des fouilles du sanctuaire de Cybèle, réactualise ses observations et conclusions sur les modes de construction et sur la chronologie des édifices lyonnais à arases de briques (Audin, 1972). Une des références pour situer l'apparition en Gaule de ce mode de construction au II $^{\mathrm{e}} \mathrm{s}$. a été et est encore l'aqueduc du Gier, dont on a placé la construction sous le règne d'Hadrien, depuis la découverte de la pierre de Chagnon (CIL XIII, 1623), à la fin du XIX ${ }^{\mathrm{e}} \mathrm{s}$. 
Cette datation, comme celle du "temple de Cybèle", a servi de base à l'établissement d'une chronologie: l'aqueduc daté de 120-130 comporte des arases doubles, comme le théâtre reconstruit à l'époque d'Hadrien, le "temple de Cybèle", daté de 160 par la découverte à proximité, en 1704, d'un autel taurobolique dédié le 9 décembre 160 (CIL XIII, 1751), présente, lui, des arases triples. C'est pratiquement ce seul critère qui a permis d'avancer la date de 160 pour l'édification de l'odéon (Audin, 1972). Bien que A. Audin ait apporté lui-même des restrictions à un système trop simple pour être totalement crédible ${ }^{1}$, celui-ci a été d'une grande influence pour la chronologie des grands monuments.

Notre propos n'est pas de remettre en cause la date de ces édifices, en particulier de l'odéon et du "temple de Cybèle", pour lesquels, en l'absence de sondages stratigraphiques, nous n'avons aucun élément nouveau à apporter ${ }^{2}$. Il s'avère cependant que la chronologie proposée pour l'apparition en Gaule de cette technique, longuement utilisée en Italie depuis le $\mathrm{I}^{\mathrm{er}} \mathrm{s}$., est tout à fait contestable et se trouve contredite par l'existence de bâtiments bien datés du $\mathrm{I}^{\mathrm{er}} \mathrm{s}$. qui présentent ce mode de construction.

Il faut rappeler tout d'abord que l'utilisation des briques dans les maçonneries se rencontre dès la période augustéenne et $\mathrm{A}$. Audin signale l'emploi de briques dans les embrasures du théâtre, en lit double ou triple ainsi que des claveaux de briques dans les arcs (Audin, 1972, p. 39). Mais il s'agit là de châ̂nages qui ne se prolongent que de 50 à $75 \mathrm{~cm}$ sur la face du mur. Un système équivalent se rencontre dans les boutiques antérieures au "temple de Cybèle" (fig. 1) actuellement datées de l'époque d'Auguste (Audin, 1985), mais que P. Wuilleumier avait autrefois attribuées au II $^{\mathrm{F}} \mathrm{s}$. en s'appuyant précisément sur la présence de lits de briques doubles (Wuilleumier, 1951 , p. 58$)^{3}$ !

Pour Lyon même, l'édifice le plus ancien où apparaissent des arases de briques continues est le

1 "Toutefois il ne convient pas, comme on a eu tendance a le faire, d'accorder trop d'importance au nombre de rangs de briques des arases" (Audin, 1972, p. 43).

2 Il faut signaler toutefois que nous venons de commencer de nouvelles fouilles a l'emplacement du "sanctuaire de Cybèlen, et on peut espérer que celles-ci apporteront d'ici peu des éléments nouveaux pour la datation et l'interprétation des différents édifices inclus dans ce secteur.

3 "Les boutiques nous ont paru posterieures (au théâtre) : on retrouve leur double rang de briques dans le grand mur du forum qu'une tradition médiévale attribue à Trajan ou Iladrien et dans l'aqueduc de Pilat, imputable à ce dernier empereur".

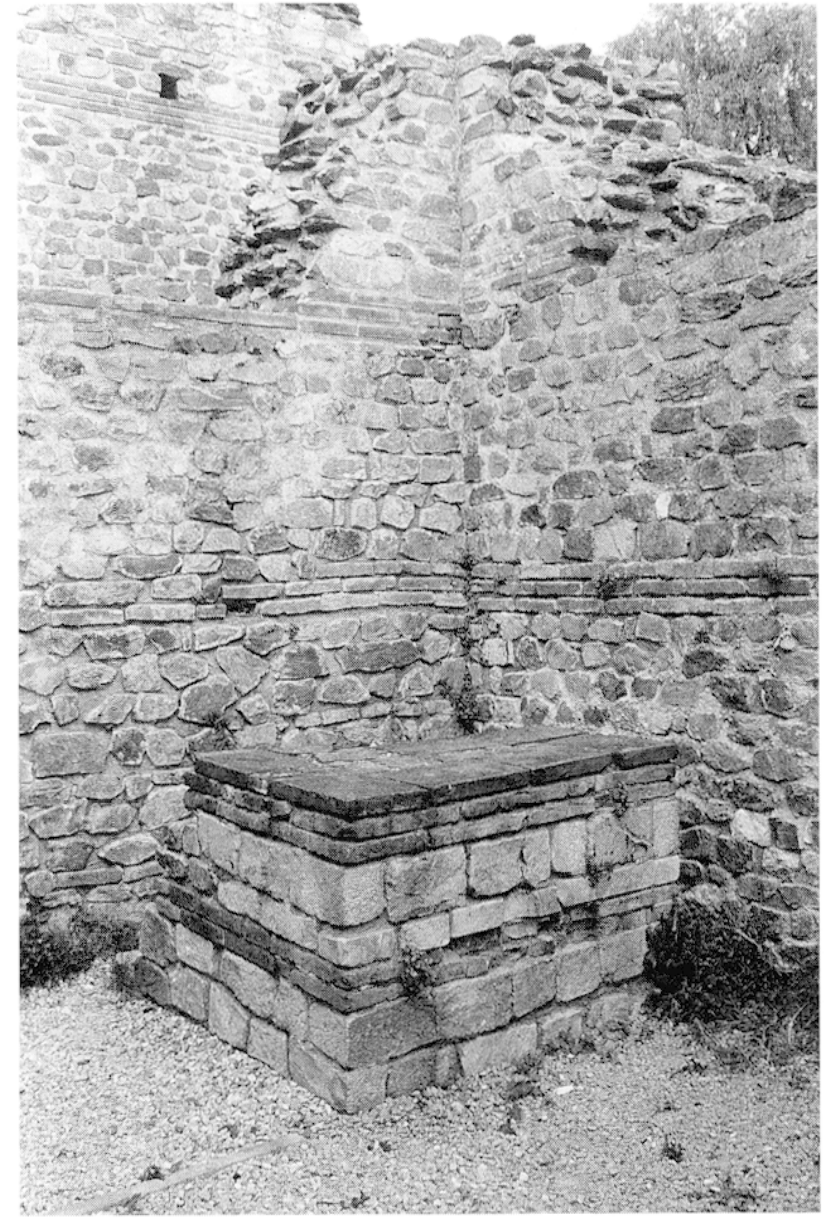

Fig. 1 - "Sanctuaire de Cybèle" (Lyon). Alvéole de la façade orientale; murs à triple rangs de briques du "sanctuaire" et mur à arases doubles des boutiques augustèennes (le rang supérieur résulte d'une restauration).

cryptoportique du sanctuaire du culte impérial découvert sur le plateau de la Sarra, dans les fouilles du Verbe Incarné (Lasfargues, Le Glay, 1980) Mandy, 1983; 1987).

Les murs du cryptoportique présentent des chainages de briques doubles ou triples (fig. 2) ${ }^{4}$. La fouille de cet ensemble a permis de dater la construction de lédifice vers les années 20 après J.-(X.

Le second édifice lyonnais du $\mathrm{I}^{\mathrm{rr}} \mathrm{s}$. à arases de briques est l'édifice thermal découvert en 1975 rue des Farges (I)esbat el alii, 1977). Il s'agit de grands thermes dont les murs présentent des arases de briques simples, doubles ou triples. L’édifice a été très ruiné et peu de murs en élévation ont été conservés. Pour certains, la destruction jusquau premier

4 Cf. Terbe Incarné. Rapport de fouilles 1979, p!. XIII. Nous remercions chaleureusement 13. Mandy de nous avoir autorise à utiliser ce document inédit. 
niveau darases de briques ne permet pas de dire si celles-ci étaient doubles ou triples. Ces thermes comportaient cependant des sous-sols qui ont été conservés et présentent des élévations de plusieurs mètres. Ainsi le couloir d'accès aux caves de l'aile ouest montre une construction en petit appareil de granite avec arases de briques dont la disposition était encore inconnue sur les monuments lyonnais, association d'arases simple, doubles et triples (fig. 3). Les caves elles-mêmes présentent la particularité d'avoir des assises doubles dans les murs latéraux et une double rangée au centre du berceau de la voûte. La fouille de lédifice a montré que ces sous-sols appartenaient à la première phase de construction, datable des années 50-60 après J.-C. Un autre élément contemporain des thermes est le grand mur de soutenement bordant à l'ouest la palestre des thermes et construit dans le même matériau que ceux-ci. Il présente des arases doubles (fig. 4). Dans le courant du II"s., toute la partie sud de ce mur (repéré sur $70 \mathrm{~m}$ de longueur) fut reconstruite toujours avec des arases de briques doubles.

Il nous faut encore signaler la découverte, à Vienne cette fois, de constructions à arases de briques antérieures au $\mathrm{II}^{\mathrm{P}} \mathrm{s}$. La première a été signalée dans les fouilles du "temple de Cybèle" (Pelletier, 1976 , p. 118). Il s'agit de deux murs, de $1,40 \mathrm{~m}$ et $1,20 \mathrm{~m}$ de large, avec des arases de briques triples, espacées de $1,17 \mathrm{~m}$, qui ont pu être datés de l'époque tibérienne.

Récemment à Saint-Romain-en-Gal, un sondage dans le portique de la voie est (voie 3 ) a révélé l'existence d'une arase de briques dans le mur du portique édifié au début du $\mathrm{I}^{\mathrm{er}} \mathrm{s}$. (fig. 5).
Ces exemples montrent que ce mode de construction ne peut ètre considéré comme un indice chronologique probant, pas plus qu'on ne peut tirer argument du nombre d'arases pour préciser la date : le mur de la rue des Farges est reconstruit au $\mathrm{II}^{\mathrm{e}} \mathrm{s}$. avec le même nombre d'arases et les thermes présentent une alternance de un, deux et trois rangs. C'est d'ailleurs cette disposition, inconnue dans le théâtre ou l'odéon, qui a été invoquée pour rapporter la construction de l'édifice à l'époque de Marc-Aurèle (Audin, 1976). On voit par là les dangers d'une chronologie fondée sur un tel système.

Par ailleurs, il convient d'exposer des doutes quant à la datation de l'aqueduc du Gier, pierre angulaire de la chronologie lyonnaise des édifices à rangs de briques (fig. 6).

Jusqu'à la découverte de la pierre de Chagnon en 1887, cet aqueduc était considéré comme une création de l'empercur Claude. Cette datation reposait sur la découverte au $x$ III $^{\mathrm{e}} \mathrm{s}$. de tuyaux de plomb marqués au nom de cet empereur à une altitude accessible par ce seul aqueduc. La découverte de la pierre de Chagnon a remis en cause cette datation, sans que la date de l'inscription ne constitue une preuve suffisante en soi (Turcan, 1978). La boucle de Chagnon constitue en fait une dérivation du tracé principal et a été considérée par plusieurs auteurs comme une construction postérieure, en particulier par Montauzan (1909, p. 210).

D'autres découvertes sont venues depuis lors grossir le dossier et poser sur de nouvelles bases la discussion sur la chronologie de cet aqueduc.

La datation de cet aqueduc au $\mathrm{II}^{\mathrm{e}} \mathrm{s}$. avait été un argument pour placer à la même époque l'urbanisa-

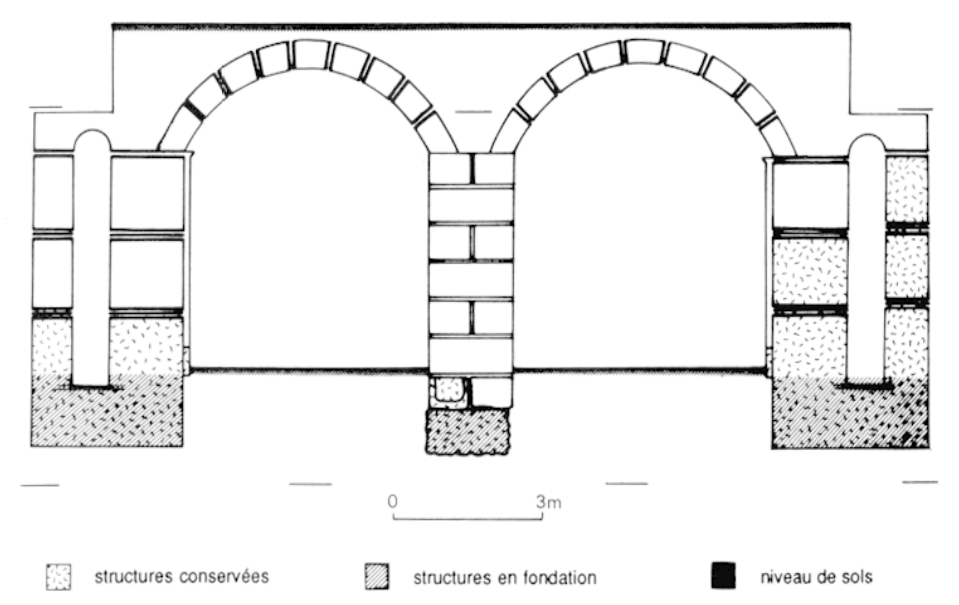

Fig. 2 - Sanctuaire du culte impérial du Verbe Incarné (Lyon). Reconstitution du cryptoportique. 

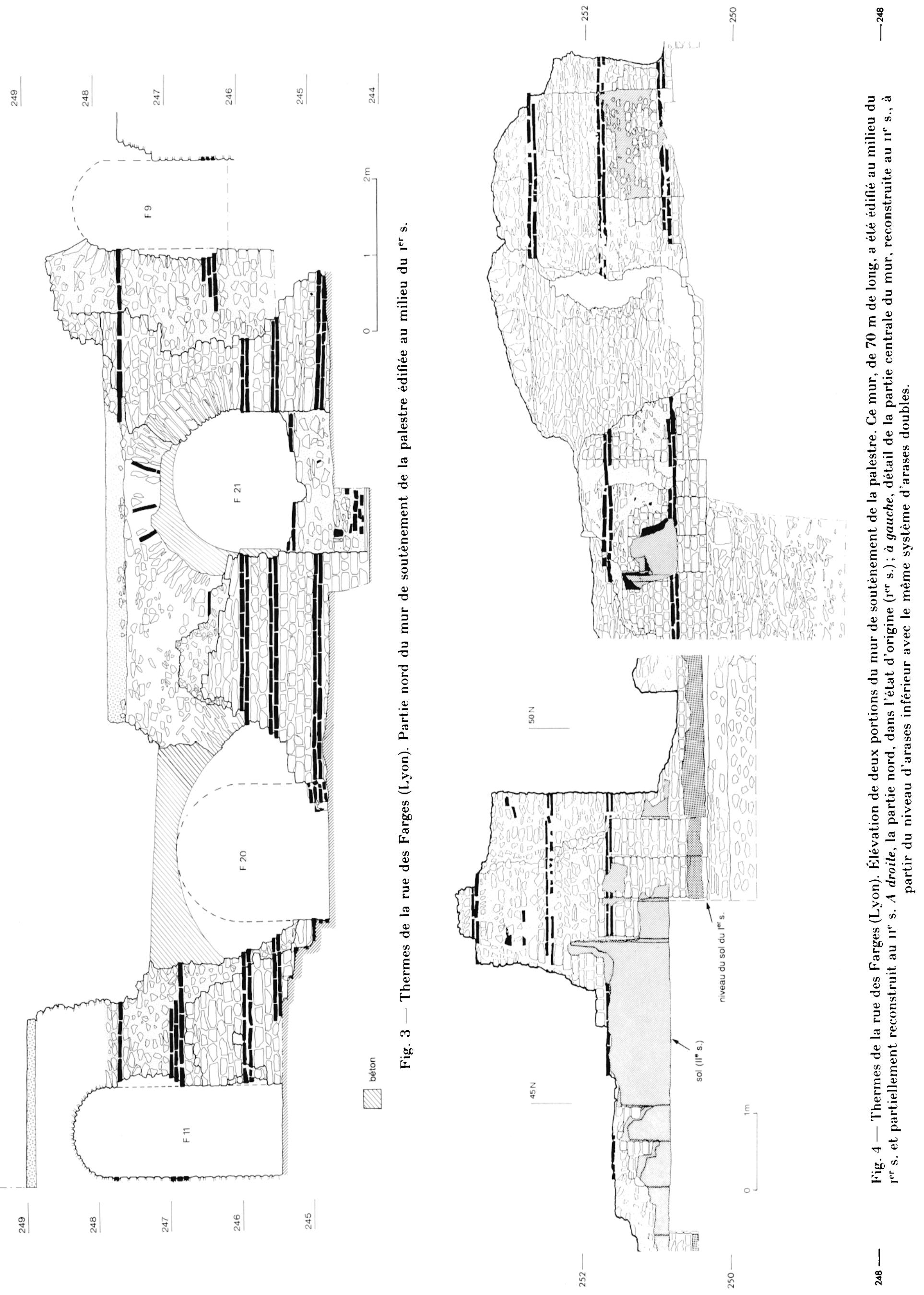


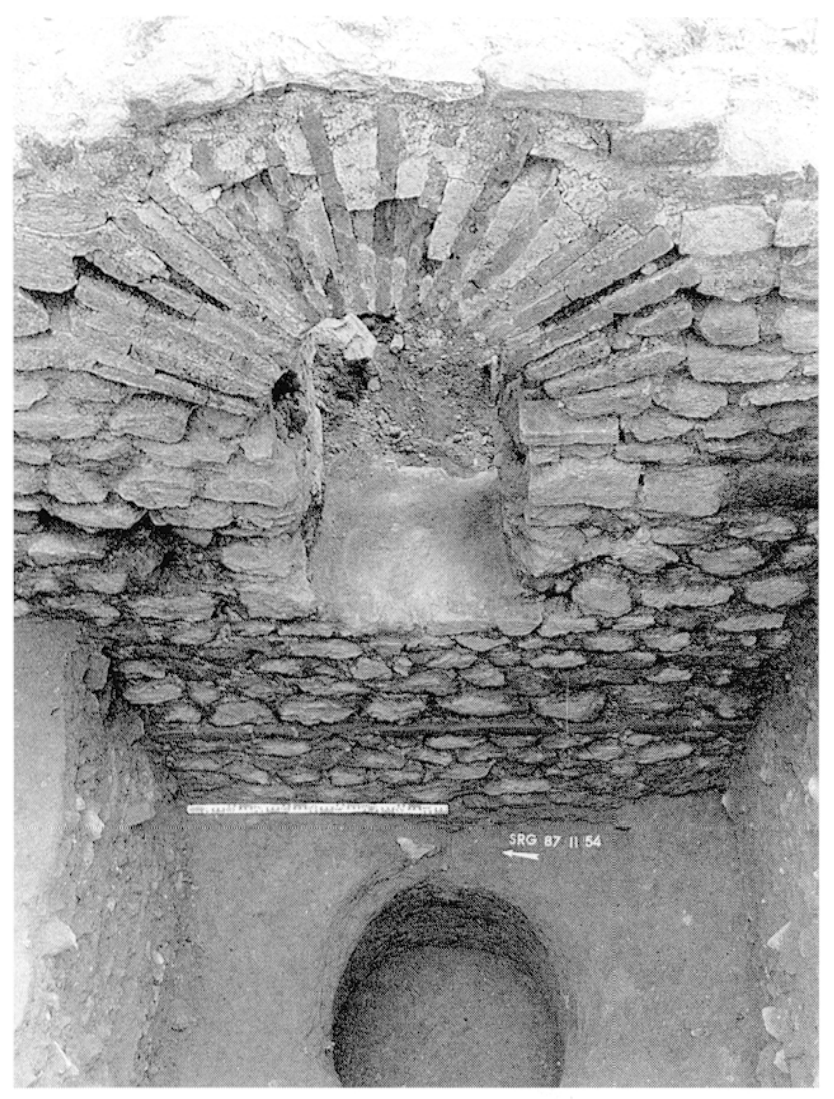

Fig. 5 - Saint-Romain-en-Gal (Rhône). Mur à chainage de briques du début du $\mathrm{I}^{\text {er }} \mathrm{s}$. tion du plateau de la Sarra jusque-là privé d'eau. Les fouilles du Verbe Incarné ont cependant montré que le quartier était urbanisé dès la fin du $\mathrm{I}^{\mathrm{er}} \mathrm{s}$. avant J.-C. et que les travaux de réaménagement du grand temple vers les années 50-60 avaient été accompagnés d'une réfection du système d'évacuation des eaux avec l'aménagement de grands collecteurs. Cet aménagement peut traduire de nouveaux besoins du quartier, corollaires à l'installation d'une arrivée d'eau abondante. Cette hypothèse des fouilleurs a d'ailleurs été reprise à son compte par D. Lavrut (1984). Cet argument insuffisant en soi a étè cependant conforté par une autre découverte. Il s'agit de celle d'une fontaine datée de Claude par l'inscription qui la surmontait (Delaval, 1989). L'altitude de cette fontaine, cotée $283 \mathrm{~m}$, implique qu'elle ne pouvait être alimentée que par l'aqueduc du Gier. Une telle chronologie pour cette construction ne paraît pas choquante. En effet, de par sa longueur et surtout la nécessité des ouvrages d'art, en particulier des siphons, qui représentent, rappelons-le, $15000 \mathrm{t}$ de plomb, la construction de cet aqueduc constitue sans

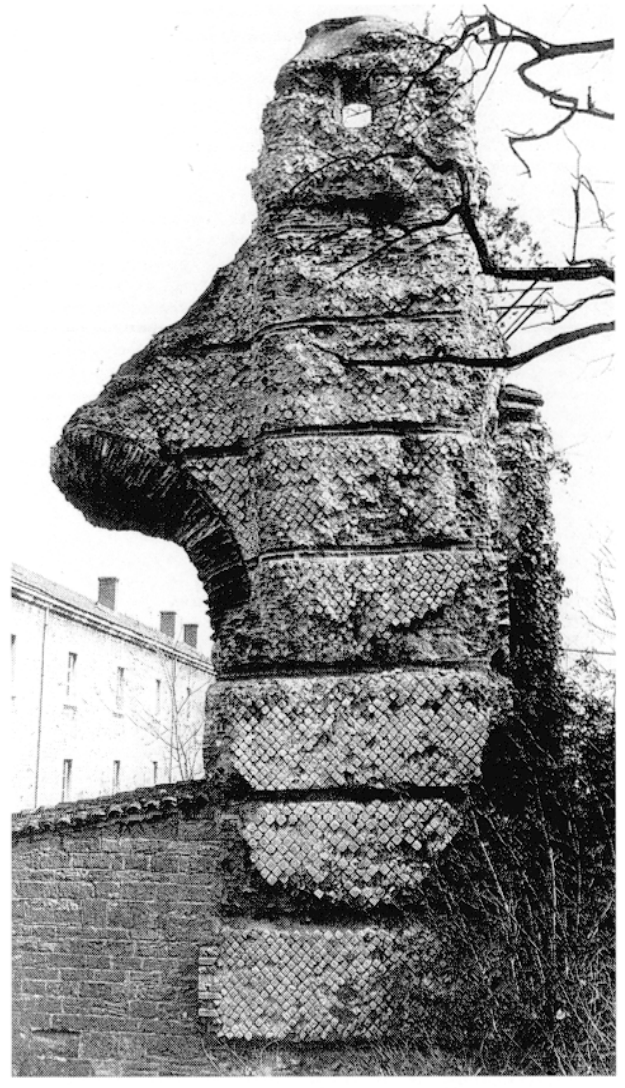

Fig. 6 - Aqueduc du Gier dans le fort Saint-Irénée (Lyon). Pile du réservoir de chasse du syphon.

nul doute la plus grande réalisation édilitaire jamais entreprise à Lyon. Que cette œuvre ait été due à la munificence de Claude, dont c'était la ville natale, paraîtrait dans la logique des choses.

Quelle que soit la datation de l'aqueduc luimême, le système établi par A. Audin en se fondant sur la chronologie des aqueducs comporte une incohérence de taille. Il s'avère en effet que l'aqueduc de la Brévenne, que l'on datait traditionnellement de Claude à la suite de Montauzan (1909), comporte précisément des arases de briques à son arrivée à Lyon. La restauration et le nettoyage des arcades de cet ouvrage, à l'extrémité de la rue des Aqueducs au Point du Jour, ont remis au jour des piles du rampant du réservoir de fuite, cachées depuis de nombreuses années sous le lierre (fig. 7). Celui-ci comporte des arases de briques triples séparées par 13 rangs de moellons.

On voit donc la contradiction qui existait soit à dater $d u{ }^{\text {er }} \mathrm{s}$. cet ouvrage utilisant une technique qui n'apparaîtrait qu'au ${ }^{2}{ }^{\mathrm{e}}$ s., soit à dater du II $^{\mathrm{e}} \mathrm{s}$. une technique déjà utilisée au $\mathrm{I}^{\mathrm{er}} \mathrm{s}$. 


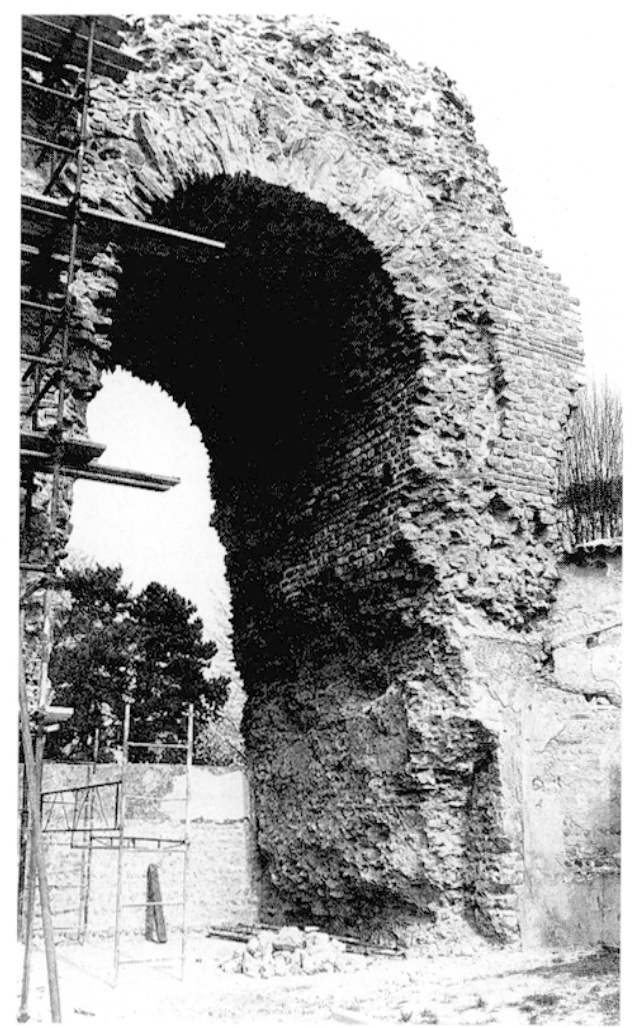

Fig. 7 - Aqueduc de la Brévenne à son arrivée rue des Aqueducs (Lyon). Pile du réservoir de fuite.
Tout ceci démontre qu'il ne reste plus grandchose des éléments sur lesquels avait été fondée la chronologie de la plupart des édifices lyonnais, mais aussi des édifices à arases de briques en Gaule. Non seulement la chronologie des monuments lyonnais est entièrement à reconsidérer mais encore la chronologie proposée pour l'utilisation de l'opus mixtum. Il est indéniable que cette technique a été utilisée en Gaule dès le $\mathrm{I}^{\mathrm{er}} \mathrm{s}$. de notre ère, même si son usage y est moins répandu qu'aux périodes plus tardives. Il n'y a là, somme toute, rien de surprenant et il était plus difficile d'expliquer qu'une technique de construction utilisée en Italie dès la fin du $\mathrm{I}^{\text {er }} \mathrm{s}$. avant J.-C. n'ait èté introduite en Gaule qu'un siècle plus tard.

\section{Armand Desbat}

\section{Origine des documents}

C. Becker (fig. 2); A. Desbat (fig. 1, 6, 7); équipe archéologique de Saint-Romain-en-Gal (fig. 5); D. Tavernier (fig. 3); D. Tavernier et M.-N. Baudran (fig. 4).

\section{BIBLIOGRAPHIE}

Adam J.-P.

1984 : La construction romaine. Matériaux et techniques, Paris, Picard, 368 p., 746 fig.

Audin A.

1972 : Techniques de construction à Lugdunum, Bulletin des Musées et Monuments Lyonnais, V, 3, p. 37-48.

1976 : L'édifice municipal des Minimes, Notes d'Épigraphie et d'Archéologie Lyonnaise, Lvon, p. 55-60.

1985 : Dossier des fouilles du sanctuaire lyonnais de Cybèle et de ses abords, Gallia, 43, p. 81-126.

Delaval E.

1989 : Une fontaine gallo-romaine alimentée par l'aqueduc du Gier découverte à Lyon (Clos du Verbe incarné à Lyon), Revue Archéologique de l'Est el du Centre-Est, 40, 2, p. 229-242.

Desbat A.

1984 : Les fouilles de la rue des Farges à Lyon (1975-1980), Lyon, 107 p., 121 fig.

Desbat A. et alii

1977: Les thermes de la rue des Farges, une découverte récente à Lyon, Archéologia, 111, p. 6-15.

Jeancolas L.

1983: Présentation des aqueducs antiques de Lyon. Journée d'étude sur les aqueducs romains, Paris, p. 179-197.
Lasfargues J., Le Glay M.

1980 : Découverte d'un sanctuaire municipal du culte impérial à Lyon, Comptes rendus de l'Académie des Inscriptions et BellesLellres, Paris, p. 394-414.

\section{Lavrut D.}

1984 : La datation de l'aqueduc du Gier, Cahiers d'Hisloire, 29, 1, p. $47-58$.

Mandy B.

1983 : Le quartier antique du Verbe Incarné, Dossiers Hisloire el Archéologie, 78, p. 23-26.

1987 : Le forum de Lyon : ètat de la question, in : Los Foros romanos de las provincias occidentales, Madrid, p. 179-183.

Montauzan C. Germain de

1909 : Les aqueducs antiques de Lyon, Paris.

Pelletier A.

1976 : Construction augusteenne et dépotoir tibérien dans le sanctuaire métroaque de Vienne (Isère), Revue Archéologique de Narbonnaise, 9, p. 115-142.

\section{Turcan R.}

1978 : Note sur la pierre de Chagnon, Bulletin de Liaison de la Direction des Antiquilés IIistoriques Rhône-Alpes (Hommage à Louis Jeancolas), 8, p. 67-71.

Wuilleumier P.

1951 : Fouilles de Fourvière à Lyon, $4^{\mathrm{e}}$ suppl. à Gallia, Paris, Éd. du C.NRS, 87 p., 8 fig., 8 plans, $X X$ pl. 